Sharif University of Technology
Scientia Iranica
SCIENTIA
IRAN ICAA

\title{
On the evolutionary characteristics of the acceleration records generated from linear time-variant systems
}

\author{
Z. Waezi and F.R. Rofooei* \\ Department of Civil Engineering, Sharif University of Technology, Tehran, Iran.
}

Received 2 April 2016; received in revised form 2 July 2016; accepted 29 August 2016

\author{
KEYWORDS \\ Non-stationary \\ records; \\ Ground motion \\ simulation; \\ Linear time variant; \\ Zero crossing; \\ Positive minimum and \\ negative maximum; \\ Lower-level crossings.
}

\begin{abstract}
This paper discusses the time-varying characteristics of the output signals resulted from Linear Time Variant (LTV) systems using level-crossing properties. These systems, used for generating synthetic records based on a target record, contain two identification processes: 1) amplitude modulating function; and 2) time-varying Impulse Response Function (IRF) parameters. To track the IRF parameters efficiently, the zerocrossing and positive minima/negative maxima cumulative curves are usually utilized as the measures of the instantaneous spectral power. Using spectral moments, analytic relations for zero-crossing and positive minima/negative maxima cumulative count are developed with respect to the IRF's evolutionary power spectrum. In order to investigate the low-frequency components of the output records efficiently, the zero-crossing rate of the velocity and the displacement records are tracked and rigorous analytic equations for their descriptions are developed. Some of the issues concerning application of the developed equations are explored using two LVT models, and the limitations of the proposed procedures are explained. Through introduction of new compatibility measures, the necessity of implementing Monte Carlo simulations would no longer be needed, and it would be possible to generate synthetic acceleration records with desirable evolutionary characteristics with much shorter computation time.
\end{abstract}

(C) 2017 Sharif University of Technology. All rights reserved.

\section{Introduction}

Considering the growing tendency toward nonlinear dynamic analyses of structures and sensitivity of these methods to ground motion excitation [1], the existing database of the recorded accelerograms does not fulfill the required variability among ground motions used [2]. Evaluating the reliability of the structures subjected to ground motion excitations in the regions without sufficient recorded data requires careful simulation of earthquake accelerograms [3]. Contrary to the physical

*. Corresponding author. Tel./Fax: +982166164233 E-mail addresses: waezi@mehr.sharif.ir (Z. Waezi); rofooei@sharif.edu (F.R. Rofooei)

doi: $10.24200 /$ sci. 2017.4254 models which are based on modeling the physics of the earthquake phenomenon [4], the stochastic methods have been used for years to regenerate the recorded ground motions from signal processing point of view. Some believe that deterministic physical methods are more reliable for lower frequencies in heterogeneous models due to the existing difficulties in accurately representing the incoherency of source radiation and wave propagation at higher frequencies [4] and the lack of site's soil information [5]. However, other researchers have shown the efficiency of these models for frequencies up to $10 \mathrm{~Hz}$ [6]. The stochastic methods can provide enough accuracy and flexibility for largescale performance-based design applications. Because of the importance of the effects of dispersion at high frequencies, most of the stochastic simulations are able to predict the ground motion at frequencies greater 
than $1 \mathrm{~Hz}$ [7]. In general, when the number of strongground motion recordings is limited with good seismological network data available, application of stochastic methods could relate the physics of the earthquakes to the wave propagation-related issues through simple relations [8]. Despite numerous studies on engineering models of earthquake motion, there is still a lack of direct experimental evidence confirming stochastic or deterministic nature of the earthquake phenomenon [3].

There are essentially two types of stochastic ground-motion models: 'source-based' and 'sitebased' [9]. Although the source-based models have the benefit of using the physical parameters obtained through simulation processes, these parameters vary significantly from region to region. In the absence of detailed seismological information, or in the case of not using that information, the site-based models are more applicable where the number of instrumental recordings is limited [10]. Due to the capability of the stochastic methods in generating high-frequency signals [11] and availability of fast computers, pure "physical models" have evolved to the "hybrid models" using stochastic methods for scenario-based earthquake simulation [12].

The development of the time-frequency methods to extract the evolutionary characteristics of the signals has made a great impact on the identification of the records' characteristics and replication of their results. The ease of use and capability to regenerate recorded data are essential factors for a method to be implemented in the process of designing structures subjected to earthquake excitation.

According to the time variation of the amplitude and the frequency content, an earthquake accelerogram as a signal can be classified either as a stationary or non-stationary realization. The amplitude nonstationarity is defined as the change in the amplitude or intensity of the acceleration record versus time, while the frequency non-stationarity indicates the change of its "instantaneous power spectrum" [9]. Contrary to the amplitude non-stationarity, it is more difficult to simulate or even capture the frequency non-stationarity of the signals. Accurate detection of the frequency content evolution demands utilization of complex timefrequency distributions (such as quadratic distributions) or multi-resolution analyses (such as wavelet or Hilbert-Huang transforms). On the other hand, the proper representation of these variations requires a considerable amount of data.

Frequency non-stationarity of the signals results from the dynamic nature of the ground motion that is mainly due to the faster propagation speed of highfrequency waves in the soil media [2]. Many studies indicate the significance of the frequency content change on the seismic-induced linear or nonlinear response of structures [13,14]. The coincidence of stiffness degradation together with the arrival of low-frequency surface waves could lead to disastrous results and even collapse of these structures [15]. Some approaches have been introduced for considering the non-stationarity of the records, especially in the frequency domain, with most of them having a large number of parameters involve $[10,16,17]$.

Rezaeian and Der Kiureghian [9,18] presented a method for record generation based on matching the zero crossing and also positive minimum, negative maxima (pos. min./neg. max.) of the recorded and ensemble of the generated accelerograms. In spite of the simplicity of the proposed method, the spectral responses of the generated accelerograms deviate from those of original records for the long-period region of the spectrum.

Moreover, the results show that for some records, the methods suggested by Rezaeian and Der Kiureghian $[9,18]$ do not lead to a suitable amount of damping values for the proposed model since the rates of pos. min./neg. max. are less than those obtained for the values of $\xi_{f}$ (damping ratio of LTV filter) from 0 to 1. Figure 1 shows the above problem for the Northridge 1/17/94, Sandberg-Bald MTN accelerogram. It should also be emphasized that the frequency and damping ratios of a high-pass filter, i.e. $f_{c}$ and $\xi_{c}$, that are used to eliminate unwanted low-frequency power spectral amplitudes greatly affect the low-frequency response spectrum region. Furthermore, the assumption of the linear variation of the frequency filter with time leads to the 2nd order polynomial cumulative zero-crossing curve which is not the case for some records.

This paper introduces a new method for stochastic generation of a non-stationary acceleration record based on the zero-crossing properties of the acceleration, velocity, and displacement records. A high-pass filtered, time-varying double-frequency model with

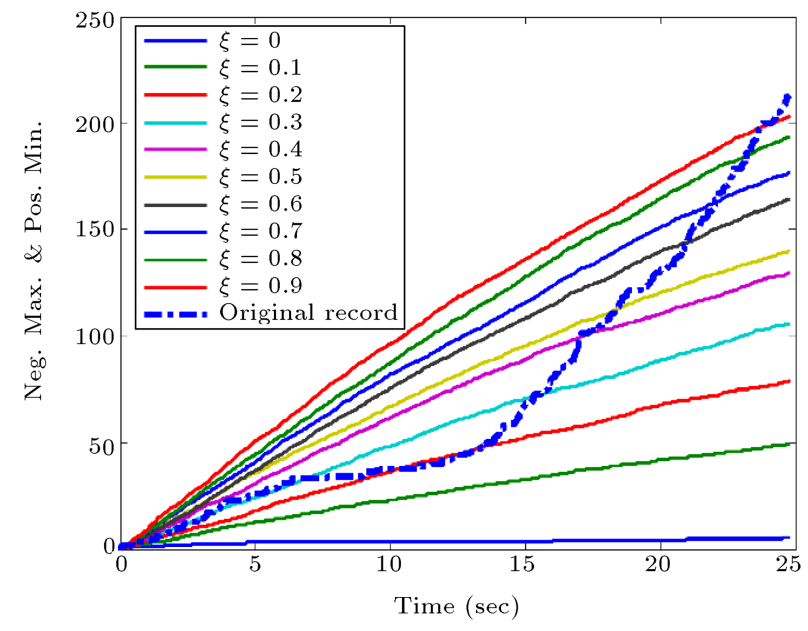

Figure 1. The comparison of the pos. min./neg. max. curves for the Northridge 1/17/94, Sandberg-Bald MTN records with those obtained from $[9,18]$. 
time-variant parameters is introduced to develop a frequency-wise, non-stationary process. The proposed model does not depend on sophisticated mathematical transformation and uses time-domain characteristics of the records to simulate seismic signals.

In the following, the concept of non-stationary records, Linear Time-Variant (LTV) systems, and method of level crossing for identification of the model parameters will be briefly described. Then, the proposed LTV model as well as the procedure for using all this information for synthesizing the earthquake records will be explained. Finally, the suggested method for the simulation of ground motions according to a scenario case will be discussed.

\section{Basic definitions}

\subsection{Frequency and amplitude non-stationarities}

The most important step in regenerating the synthetic, yet realistic, accelerograms is the identification of the properties of the recorded ground motions. The idea of an "instantaneous dominant frequency" for non-stationary signals has always been appealing to scientists because of its easier interpretation. One of the earliest approaches towards this purpose has been defined through re-writing a signal as a multiplication of amplitude and a harmonic part. Since its introduction by Gabor, one can easily transform a real signal, like $x(t)$, to "analytic" complex signal $x^{+}(t)$ using Hilbert transform without loss of information [19] as in the following:

$$
x^{+}(t)=x(t)+i v(t)
$$

where $i$ is the imaginary unit. Here, $v(t)$ is called Hilbert transform of $x(t)$ and is defined as:

$$
v(t)=\frac{1}{\pi} P . V .\left(\int_{-\infty}^{\infty} \frac{x(\tau)}{t-\tau} d \tau\right),
$$

where P.V. stands for the principal value of the integral. The analytic signal can be described in a canonical form by its amplitude $A(t)$ and phase angle $\phi(t)$ defined as follows:

$$
A(t)=\left|x^{+}(t)\right|, \quad \phi(t)=a \tan \frac{v(t)}{x(t)} .
$$

Using the above formula, it is possible to define the Instantaneous Frequency (IF) of a signal as $\omega(t)=$ $d \phi / d t$ as long as $A(t)$ has distinct frequency band from those of harmonic part. Huang et al. [20] introduced the Empirical Mode Decomposition method (EMD) to decompose every non-stationary signal into several single-component sub-signals called Intrinsic Mode Function (IMF). Then, it is easy to estimate the instantaneous frequency of every component through Hilbert transform, which is commonly known as Hilbert-Huang Transform (HHT). Huang also introduced the normalized HHT method to normalize IMFs as Frequency Modulated (FM) as well as Amplitude Modulated (AM) signals [21]. According to a weighting function based on each IMF's amplitude, the obtained IF from each FM can be averaged to estimate the Weighted Instantaneous Frequency (WIF) of the signal as follows:

$$
\mathrm{WIF}(t)=\frac{\sum_{j=1}^{n} A_{j}(t) I F_{j}(t)}{\sum_{j=1}^{n} A_{j}(t)},
$$

where $A_{j}$ and $I F_{j}$ represent the amplitude and instantaneous frequency of the $j$ th IMF. One of the most important advantages of the canonical form of a signal is the ability to use spectral moments for its description. The Fourier transform of the analytic signal, $X^{+}(\omega)$, is:

$$
X^{+}(\omega)=2 U(\omega) X(\omega)
$$

where $X(\omega)$ and $U(\omega)$ are the Fourier transforms of the signal and unit step function, respectively. General mean frequency, $\bar{\omega}_{x}$, and mean bandwidth of signal, $\bar{\sigma}_{x}^{2}$, can be defined as follows:

$$
\begin{aligned}
& \bar{\omega}_{x}=\frac{1}{2 \pi S_{x}} \int_{0}^{\infty} \omega\left|X^{+}(\omega)\right|^{2} d \omega, \\
& \bar{\sigma}_{x}^{2}=\frac{1}{2 \pi S_{x}} \int_{0}^{\infty}\left(\omega-\bar{\omega}_{x}\right)^{2}\left|X^{+}(\omega)\right|^{2} d \omega,
\end{aligned}
$$

where:

$$
S_{x}=\frac{1}{2 \pi} \int_{0}^{\infty}\left|X^{+}(\omega)\right|^{2} d \omega=\int_{-\infty}^{\infty}\left|x^{+}(t)\right|^{2} d t .
$$

On the other hand, when the response of the systems subjected to these signals is of concern, the estimation of their time-varying power spectrum is of greater importance for which two classic approaches are defined by Page [22] and Priestley [23]. Introducing the "instantaneous power spectrum", Page extended the concept of energy density of stationary processes to the non-stationary ones through the change of spectral power in time. For a non-stationary process $\{a(t)\}$, "the instantaneous power spectrum" $\rho(t, f)$ at time $t$ for frequency $f$ is defined as follows:

$$
\rho(t, f)=2 \int_{0}^{\infty} a(t) a(t-\tau) \cos (2 \pi f \tau) d \tau .
$$

On the other hand, Priestley's Evolutionary Power Spectral Density (EPSD) can rigorously describe the energy distribution of a random process in the vicinity of time $t$. If a non-stationary process $\{a(t)\}$ can be written in the following form:

$$
a(t)=\int_{-\infty}^{\infty} m(t, \omega) e^{i \omega t)} d z(\omega)
$$


where $m(t, \omega)$ is a complex modulating function, $d z(\omega)$ is a complex-valued random process, and its EPSD $S(t, \omega)$ can be defined as follows:

$$
S(t, \omega)=|m(t, \omega)|^{2} d \mu(\omega)
$$

where $E\left[|d z(\omega)|^{2}\right]=d \mu(\omega)$ represents the PSD of the stationary random process used in Eq. (9). Saragoni and Hart [24] defined the EPSD for finite-time regions, while Liu [25] developed a method to calculate the EPSD for a better demonstration of the nonstationarity in earthquake accelerograms. Considering the evolutionary model of Priestly in the form of Eq. (9), the uniformly-modulated model is one of the primary ones to generate stochastic ground motions as follows:

$$
a(t)=m(t) s(t)
$$

where $a(t), s(t)$ and $m(t)$ represent ground acceleration process, stationary process, and a real-valued modulating function, respectively. The models introduced by Amin and Ang [26] and Shinozuka and Sato [27] belong to this category of stochastic simulation of nonstationary signals. Using a time-scale modulator, Yeh and Wen's model [14] also considers the same form as in Eq. (11), but adjusts the dominant frequency of the ground motion. Conte and Peng [13] modeled the earthquake records using a bundle of waves with different arrival times using the evolutionary model of Priestly. Liang et al. [28] used the Priestley's evolutionary model to derive a cosine series formula to simulate the non-stationary Gaussian processes based on spectral representation. They have proposed three methods to estimate the evolutionary energy content using short-time Fourier transform, wavelet transform, and Hilbert-Huang transform. Yamamoto and Baker used wavelet transform to approximately estimate the evolutionary spectrum of the earthquakes [16]. They calibrated the parameter of wavelet transform to relate them to the ground motion parameters. Kaveh and Mahdavi used the wavelet transform and an improved optimization technique to decompose the original ground motions to several levels multiplied by a variable in order to get better spectral matching for the simulated ground motions [29]. Papadimitriou used the 2 nd order differential equation with time-variable coefficients subjected to white noise excitation [30]. In a different approach, Rezaeian and Der Kiureghian used a filtered white noise, whose non-stationarity is separated into uncorrelated amplitude and frequency non-stationarities $[9,18]$. They used the time-domain characteristics of the output signal, instead of frequency domain, to adjust the model parameters.

The amplitude non-stationarity can usually be described by a modulating or envelope function. There are different definitions for the envelope of a random process. While Rice's envelope [31] is based on the expansion of the process about a "mid-band" frequency, Dugundji used Hilbert transform to calculate the pre-envelope process corresponding to the random process, and then they estimated the envelope of the process [32]. Sgobba et al. [33] used this idea to evaluate the envelope functions of the accelerograms and approximated that with a Husid Envelope Function (HEF) according to the lognormal distribution. The envelope of Crandall and Mark [34] is estimated according to an "energy" function defined using the process and its derivative. A number of well-known modulating functions are presented in Table 1. Unlike most of these methods' fitting procedures which are based on the minimization of the Arias intensity difference between the target and simulated records, Stafford et al. [10] used the Dugundji's definition [32] to fit their proposed envelope function using a log-normal probability distribution function. Despite having more parameters, Amin and Ang's [26] piecewise model could effectively capture a wider range of amplitude nonstationarity for the recorded accelerograms. Methods based on moving average techniques that are adopted by some researchers to better consider the amplitude change are not considered in this study due to the difficulty in their application for the scenario-based record simulation since they cannot be described by an analytical expression.

\subsection{LTV systems}

A Linear Time-Invariant (LTI) SDOF system is identified by its unit Impulse Response Function (IRF) to the impulse applied on time $\tau$ represented by $h(t-\tau)$. The output of such a system subjected to the Gaussian white-noise excitation $w(\tau)$ can be estimated using the following convolution integral:

$$
f(t)=\int_{-\infty}^{t} h(t-\tau) w(\tau) d \tau
$$

However, the LTV systems are characterized by a timevarying impulse response $h(t-\tau, \tau)$ that is dependent on both impulse incidence time $(\tau)$ as well as elapsed time $(t-\tau)$. Since the nonlinear systems can be simulated as an LTV model, given that the system characteristics are well-defined, their application in the field of artificial record generation has gained much attention. An LTV system, whose output to white noise includes both types of non-stationarities, can be defined by adjusting Eq. (12):

$$
f(t)=\int_{-\infty}^{t} h(t-\tau, \tau) w(\tau) d \tau .
$$

It is easy to impose the non-stationarity on the LTI's IRF through making a set of model parameters to be the functions of impulse incidence time $(\tau)$, guaranteeing a time-varying Power Spectral Density (PSD) as 
Table 1. Some of the modulating functions used by researchers.

\begin{tabular}{|c|c|c|}
\hline Authors & Model name & Expression \\
\hline $\begin{array}{c}\text { Bolotin [35] \& Shinozuka } \\
\text { and Sato [27] }\end{array}$ & Exponential model & $q(t, \alpha, \beta, \gamma)=\alpha\{\exp (-\beta t)-\exp (-\gamma t)\} \quad \beta<\gamma$ \\
\hline Amin \& Ang 1968 [26] & Piece-wise modulating & $q(t, \alpha)=\left\{\begin{array}{l}0 \quad T_{0} \geq t \\
\alpha_{1}\left(\frac{t-T_{0}}{T_{1}-T_{0}}\right)^{2} \quad T_{0} \leq t \leq T_{1} \\
\alpha_{1} \quad T_{1} \leq t \leq T_{2} \\
\alpha_{1} \exp \left[-\alpha_{2}\left(t-T_{2}\right)^{\alpha_{3}}\right] \quad T_{2} \leq t\end{array}\right.$ \\
\hline Saragoni \& Hart 1974 [24] & Gamma function & $q(t, \alpha)=\left\{\begin{array}{l}0 \quad T_{0} \geq t \\
\alpha_{1}\left(t-T_{0}\right)^{\alpha_{2}-1} \exp \left[-\alpha_{3}\left(t-T_{0}\right)\right]\end{array}\right.$ \\
\hline Arias et al. [36] & Beta model & $m_{k}(t)=\alpha_{k}\left(\frac{t}{t_{f}}\right)^{\beta_{k}}\left(1-\frac{t}{t_{f}}\right)^{\gamma_{k}}$ \\
\hline Kiureghian \& Crempien [15] & Piece-wise linear & $m_{k}(t)=\alpha_{k}\left(t-t_{i}\right)+\beta_{k i}, \quad t_{i}<t<t_{i+1}$ \\
\hline Yeh [37] & - & $A t^{B}\left(D+t^{E}\right)^{-1} e^{-C t}$ \\
\hline Stafford et al. [10] & $\begin{array}{l}\text { The Husid Envelope } \\
\text { Function (HEF) }\end{array}$ & $E(t)=\sqrt{\frac{4 g I_{a}}{t \sigma \pi \sqrt{2 \pi}} \exp \left(-\frac{(\ln (t)-\mu)^{2}}{2 \sigma^{2}}\right)}$ \\
\hline
\end{tabular}

the IRF evolves. Nevertheless, given an Evolutionary Transfer Function (ETF), i.e. $H(\omega, \tau)$, obtained from Priestley's [23] definition of the evolutionary spectrum, one can find unit impulse function $h(t, \tau)$ as:

$$
\begin{aligned}
& h(t, \tau)=\frac{1}{2 \pi} \int_{-\infty}^{\infty} H(\omega, \tau) \exp (i \omega(t-\tau)) d \omega \\
& \text { for } t \geq \tau
\end{aligned}
$$

Thus, more complicated non-stationary signals with desired instantaneous properties may be generated using this approach. In the proposed model by [9], the ETF's dependence on parameters $\omega_{f}$ and $\xi_{f}$ determines the dominant frequency and the bandwidth of the produced records. It is obvious that using a model similar to Eq. (13) subjected to white noise results in the generation of signals whose amplitude non-stationarity is severely affected by its evolutionary spectrum. By the idea of Rezaeian and Der Kiureghian [9], it is possible to build an LTV with a separable amplitude and power non-stationarity as long as the amplitude and IRF's PSD's do not overlap:

$$
f(t)=q(t)\left\{\frac{1}{\sigma(t)} \int_{-\infty}^{t}(t-\tau, \lambda) w(\tau) d \tau\right\},
$$

where $q(t)$ and $\sigma^{2}(t)$ represent the amplitude modulating function and the variance of the integral output, respectively. Waezi and Rofooei used the following EPSD along with Eq. (15) to define an LTV compatible system for the better simulation of the near-field pulseless records [38]:

$$
\begin{aligned}
& S(\omega, t)= \\
& \frac{\left(\frac{\omega}{\omega_{f}(t)}\right)^{4}\left(1+\left(\frac{2 \xi_{g} \omega}{\omega_{g}}\right)^{2}\right)}{\left(1-\frac{\omega^{2}}{\omega_{f}(t)^{2}}\right)^{2}+\left(\frac{2 \xi_{f}(t) \omega}{\omega_{f}(t)}\right)^{2}\left(1-\frac{\omega^{2}}{\omega_{g}^{2}}\right)^{2}+\left(\frac{2 \xi_{g} \omega}{\omega_{g}}\right)^{2}} S_{0}
\end{aligned}
$$

where $\xi_{g}$ and $\omega_{g}$ are constant model parameters and $\xi_{f}$ and $\omega_{f}$ are the time-variant model parameters that change linearly in time. For the same purpose, the following EPSD as a Double-Frequency Model (DFM) is defined here to be used in Eq. (15) which has nonzero, low-frequency energy content:

$$
\begin{aligned}
& S(\omega, t)= \\
& \frac{1+\left(\frac{2 \xi_{f}(t) \omega}{\omega_{f}(t)}\right)^{2}}{\left(1-\frac{\omega^{2}}{\omega_{f}^{2}(t)}\right)^{2}+\left(\frac{2 \xi_{f}(t) \omega}{\omega_{f}(t)}\right)^{2}} \frac{1+\left(\frac{2 \xi_{g} \omega}{\omega_{g}}\right)^{2}}{\left(1-\frac{\omega^{2}}{\omega_{g}^{2}}\right)^{2}+\left(\frac{2 \xi_{g} \omega}{\omega_{g}}\right)^{2}} .
\end{aligned}
$$

For the identification of the output, only LTV dynamic systems, frequency domain, and time-domain approaches can be used. Most of the frequency-domain methods, such as Discrete Fourier Transform (DFT), Fast Fourier Transform (FFT), etc., have their own drawbacks in losing time-domain information. The time-domain methods, such as autoregressive moving average methods (ARMA) [39], have been utilized to compensate for the weakness of frequency-domain methods in the identification of the local properties of the signals.

Various time-frequency representations have been 
introduced for non-stationary signals from which ShortTime Fourier Transform (STFT), wavelet, HilbertHuang, and Wigner-Ville transforms have gained more popularity. The time-frequency distributions, e.g. Wigner-Ville distribution, use the quadratic integral transformations which lead to a better frequency track by eliminating some cross terms in the output [40]. The Empirical Mode Decomposition (EMD) algorithm can be employed along with Hilbert transform for a multiresolution analysis of non-stationary signals.

If the input and output of systems are known, adaptive algorithms may be used to determine the model parameters. The Least Mean Square method (LMS) and Recursive Least Square method (RLS) that use the steepest descent algorithm to determine the unknown model' parameters are among these methods. The applicability of such methods for the identification of LTV output-only systems is doubted due to the large computational cost. According to the Basis Expansion Model (BEM), the unknown IRF of the LTV system can be described as the response superposition of LTI systems (Single Input Multiple Output system (SIMO)), which is adopted by some researchers to better address LTV systems behavior [41].

Rofooei et al. used the averaged zero-crossing rate of the acceleration signal to identify the non-stationary Kanaii-Tajimi model's ground frequency, $\omega_{g}(t)$, while keeping the ground damping ratio, $\xi_{g}(t)$, constant [42]. Rezaeian and Der Kiureghian used the zero-crossing rate as well as the pos. min./neg. max. number of the output signals to identify system parameters $\omega_{f}$ and $\xi_{f}$ $[9,18]$.

\section{Evolutionary characteristics of the signal}

\subsection{Developing equations for level crossings of the signals}

When using HHT-based methods to extract the IF of acceleration records, the obtained WIF in some cases merely becomes a numerical average among a number of modes with a similar energy level. Thus, it does not relate to the physics of the phenomenon, and the number of modes to be considered affects the resulting IF. The zero crossing of a signal contains valuable information of the evolutionary characteristics of the signal. It is defined as the number of intersections of the signal with zero-ordinate horizontal axis in any specific time. According to Wiener-Khintchine theorem, for a stationary Gaussian stochastic discrete process, $Z_{t}$, of length $N$ with mean value, $E\left[Z_{t}\right]=\mu$, and constant covariance, there always exists a strictly ascending function, $F(\omega)$, for which one could have [43]:

$$
\gamma_{k}=\int_{-\pi}^{+\pi} \cos (k \omega) d F(\omega)
$$

where $\gamma_{k}=E\left[Z_{t} Z_{t-k}\right]$ defined for $k=0, \pm 1, \ldots$ is the autocovariance of $Z_{t}$. It can be proved that the mathematical expectation of the total number of zero crossing $E\left[Z C_{1}\right]$ is [44]:

$$
\cos \left(\frac{\pi E\left[Z C_{1}\right]}{N-1}\right)=\frac{\int_{-\pi}^{\pi} \cos (\omega) d F(\omega)}{\int_{-\pi}^{\pi} d F(\omega)},
$$

where $\omega$ is the discrete frequency changing from $-\pi$ to $\pi$. Moreover, it can be shown that for Gaussian output process obtained by the application of a linear filter $\mathcal{L}$ with transfer function $H$, the total number of zero crossings $Z C_{H 1}$ can be obtained using [44]:

$$
\cos \left(\frac{\pi E\left[Z C_{H 1}\right]}{N-1}\right)=\frac{\int_{-\pi}^{\pi} \cos (\omega)|H(\omega)|^{2} d F(\omega)}{\int_{-\pi}^{\pi}|H(\omega)|^{2} d F(\omega)} .
$$

By defining the 1 st, $2 \mathrm{nd}$, and $k$ th derivatives of discrete process, $Z_{t}$, as $\nabla Z_{t} \equiv Z_{t}-Z_{t-1}, \nabla^{2} Z_{t}=\nabla\left(\nabla Z_{t}\right)=$ $Z_{t}-2 Z_{t-1}+Z_{t-2}$, and $\nabla^{k} Z_{t} \equiv \sum_{j=0}^{k}\left(\begin{array}{c}k \\ j\end{array}\right)(-1)^{j} Z_{t-j}$, respectively, Eq. (20) can be used to determine their corresponding zero crossings which are called $Z C_{2}$, $Z C_{3}$, and $Z C_{k+1}$, respectively, as follows:

$$
\cos \left(\frac{\pi E\left[Z C_{k+1}\right]}{N-1}\right)=\frac{\int_{-\pi}^{\pi} \cos (\omega)\left(\sin \frac{\omega}{2}\right)^{2 k} d F(\omega)}{\int_{-\pi}^{\pi}\left(\sin \frac{\omega}{2}\right)^{2 k} d F(\omega)}
$$

These relations can be used to define the spectral power of any discrete signal in a procedure explained by Kedem [44]. On the other hand, for a zero mean Gaussian, twice differentiable, stationary signal $\{y(t)\}$ for $-\infty<t<\infty$, with auto-covariance function $R(\tau)$, and autocorrelation function $\rho(\tau)$, the zero crossing rate, i.e. the total number of zero crossing in unit time, can be determined according to Rice's Formula [31]:

$$
E\left[Z C_{1}\right]=\frac{1}{\pi} \frac{\sigma_{\dot{Y}}}{\sigma_{Y}}=\frac{1}{\pi}\left[\frac{\int_{0}^{\infty} \omega^{2} S(\omega) d \omega}{\int_{0}^{\infty} S(\omega) d \omega}\right]^{\frac{1}{2}}
$$

where $S(\omega), \sigma_{\dot{Y}}$, and $\sigma_{Y}$ are the power spectral density, standard deviation of $\{y(t)\}$, and $\{\dot{y}(t)\}$ processes, respectively. In this study, instead of total zero crossing, the zero crossing with a positive rate will be considered as an identification tool denoted as $Z C^{+}$. It can be easily shown that for a Gaussian signal, $Z C^{+}$is equal to the half of the total $Z C$ obtained by Eq. (22). Using a similar procedure, one can obtain $Z C^{+}$formula for a non-stationary signal, $y(t)$, with the stationary frequency content as:

$$
\begin{aligned}
Z C^{+}(t) & =\frac{\left(1-\rho_{Y \dot{Y}}(t, t)^{2}\right)^{\frac{1}{2}}}{2 \pi} \frac{\sigma_{\dot{Y}}(t)}{\sigma_{Y}(t)} \\
& =\frac{\left(1-\rho_{Y \dot{Y}}^{2}(t, t)\right)^{\frac{1}{2}}}{2 \pi} \omega_{c},
\end{aligned}
$$


where $\rho_{Y \dot{Y}}$ and $\omega_{c}$ are the correlation coefficient of the process with its time-derivative and the central frequency of the signal, respectively. Waezi and Rofooei [38] recommended the following relation for $Z^{+}$ count of signals with non-stationary power spectrum $S(\omega, \tau)$, ignoring the effect of $\rho_{Y \dot{Y}}$ due to its negligible effect on the final results and only considering the slowly-varying system parameters:

$$
E\left[Z C^{+}\right]=\int_{0}^{t} \frac{1}{2 \pi \Delta t} a \cos \left(\frac{\int_{-\pi}^{\pi} \cos (\omega) S(\omega, \tau) d \omega}{\int_{-\pi}^{\pi} S(\omega, \tau) d \omega}\right) d \tau,
$$

where $\Delta t$ is the time step. While the zero crossing of signals is related to the central frequency of the signal, the frequency bandwidth is of equal importance for some cases and can be related to the physical properties of the system too.

In the case of an LTI, SDOF system, the bandwidth of response of the system to white noise input is dependent on its damping ratio. It can be proved that the number of pos. min./neg. max. of a signal corresponds to the frequency bandwidth of the signal. For a stochastic Gaussian function of time, i.e. $a(t)$, a probability density function of the stochastic parameters can be defined as $p(\xi, \eta, \zeta)$ in which $\xi=a(t)$, $\eta=\dot{a}(t)$, and $\zeta=\ddot{a}(t)$. According to the central limit theorem, it can be stated that the distributions of $\eta$ and $\zeta$ approach the normal distribution. The second moment of this distribution can be estimated via the prescribed definitions of $\xi, \eta$, and $\zeta$ as well as the following correlation functions:

$$
\begin{aligned}
& E\left[\xi^{2}\right]=\psi_{0}, \quad E\left[\eta^{2}\right]=-\psi_{0}^{\prime \prime}, \quad E[\xi \eta]=0, \\
& E[\eta \zeta]=0, \quad E[\xi \zeta]=\psi_{0}^{\prime \prime}, \quad E\left[\zeta^{2}\right]=\psi_{0}^{(4)} .
\end{aligned}
$$

For every even number, $k$, it can be deduced that:

$$
\psi_{0}^{(k)}=(-1)^{\frac{k}{2}} \int_{0}^{\infty} \omega^{k} S(\omega) d \omega
$$

Thus, given the covariance matrix of random vector $\mathbf{X}=\left\{\mathbf{X}_{1}, \ldots, \mathbf{X}_{N}\right\}^{T}$ defined according to [31] as $M_{i j}=$ $\operatorname{cov}\left(X_{i}, X_{j}\right)=E\left[\left(X_{i}-\mu_{i}\right)\left(X_{j}-\mu_{j}\right)\right]$ where $\mu_{i}=$ $E\left(X_{i}\right)$, the multivariate normal distribution function can be described as follows:

$$
\begin{aligned}
f_{x}\left(x_{1}, \ldots, x_{n}\right)= & \frac{1}{\sqrt{(2 \pi)^{n}|\mathbf{M}|}} \exp \\
& \left(-\frac{1}{2}(\mathbf{x}-\boldsymbol{\mu})^{T} \mathbf{M}^{-1}(\mathbf{x}-\boldsymbol{\mu})\right),
\end{aligned}
$$

where $|\mathbf{M}|$ is the determinant of matrix $\mathbf{M}$ defined as follows:

$$
M=\left[\begin{array}{ccc}
\psi_{0} & 0 & \psi_{0}^{\prime \prime} \\
0 & -\psi_{0}^{\prime \prime} & 0 \\
\psi_{0}^{\prime \prime} & 0 & \psi_{0}^{(4)}
\end{array}\right] .
$$

The Probability Distribution Function (PDF) of the maxima can now be computed according to the following multivariate normal distribution:

$$
\begin{aligned}
p(x, 0, \zeta)= & (2 \pi)^{-\frac{3}{2}}|M|^{-\frac{1}{2}} \exp \left[\frac { 2 } { 2 | M | } \left(M_{11} x^{2}\right.\right. \\
& \left.\left.+M_{33} \zeta^{2}+2 M_{13} x \zeta\right)\right] .
\end{aligned}
$$

The probability of $x$ having a maximum in the time domain of $[t, t+d t]$ between $x$ and $x+d x$ is equal to:

$$
d P=-d t d x \int_{-\infty}^{0} p(x, 0, \zeta) \zeta d \zeta
$$

Integrating Eq. (30) over $a \leq t \leq b$ would lead to a number of maxima in that time period. For the total number of positive minima in the time domain $[t, t+d t]$, one can write:

$$
\text { Pos } \operatorname{Min}=d t \int_{0}^{\infty} d x \int_{0}^{\infty} p(x, 0, \zeta) \zeta d \zeta
$$

Derivation of both sides of the above equations with respect to time would yield the rate of negative maxima occurrence, which can be easily calculated using the assumed PDF described by Eq. (29):

$$
\begin{aligned}
\frac{d(\operatorname{Neg} \operatorname{Max})}{d t} & =\frac{d(\operatorname{Pos} \operatorname{Min})}{d t} \\
& =\frac{1}{4 \pi}\left(\sqrt{-\frac{\psi_{0}^{(4)}}{\psi_{0}^{\prime \prime}}}-\sqrt{-\frac{\psi_{0}^{\prime \prime}}{\psi_{0}}}\right) .
\end{aligned}
$$

Since the signal is Gaussian, it can be assumed that the number of pos. min./neg. max. is equal; thus, the total rate of pos. min./neg. max. is:

$$
\begin{gathered}
\frac{d(\text { Pos Min \& Neg Max })}{d t}=\frac{1}{2 \pi}\left(\sqrt{-\frac{\psi_{0}^{(4)}}{\psi^{\prime \prime}{ }_{0}}}-\sqrt{-\frac{\psi_{0}^{\prime \prime}}{\psi_{0}}}\right) \\
=\frac{1}{2 \pi}\left(\sqrt{\frac{\int_{0}^{\infty} \omega^{4} S(\omega) d \omega}{\left.\int_{0}^{\infty} \omega^{2} S(\omega) d \omega\right)}}-\sqrt{\frac{\int_{0}^{\infty} \omega^{2} S(\omega) d \omega}{\int_{0}^{\infty} S(\omega) d \omega}}\right),
\end{gathered}
$$

where $\psi_{0}^{(k)}=(-1)^{\frac{k}{2}} \int_{0}^{\infty} \omega^{k} S(\omega) d \omega$. Based on the foregoing discussion, Waezi and Rofooei [38] suggested the following equation for pos. min./neg. max. count for signals with non-stationary power spectrum, $S(\omega, \tau)$, considering slowly-varying system parameters in Eq. (33):

E(Pos. Min. \& Neg. Max.)

$$
\begin{aligned}
= & \int_{0}^{t} \frac{1}{2 \pi \Delta t} \operatorname{acos}\left(\frac{\int_{-\pi}^{\pi} \cos (\omega) \sin \left(\frac{\omega}{2}\right)^{2} S(\omega, \tau) d \omega}{\int_{-\pi}^{\pi} \sin \left(\frac{\omega}{2}\right)^{2} S(\omega, \tau) d \omega}\right) d t \\
& -\int_{0}^{t} \frac{1}{2 \pi \Delta t} \operatorname{acos}\left(\frac{\int_{-\pi}^{\pi} \cos (\omega) S(\omega, \tau) d \omega}{\int_{-\pi}^{\pi} S(\omega, \tau) d \omega}\right) d t .
\end{aligned}
$$




\section{Velocity and displacement zero-crossing}

Velocity and displacement time histories contain accentuated information about the power of the signal harmonics with frequencies close to zero. Having determined the velocity record's PSD, the zero crossing of the stationary Gaussian signal can be determined using the following formula provided by Rice (Eq. (22)) or Kedem (Eq. (19)):

$$
\cos \left(\frac{2 \pi E\left[V_{1}^{+}\right]}{N-1}\right)=\frac{\int_{-\pi}^{\pi} \cos (\omega) S_{V}(\omega) d \omega}{\int_{-\pi}^{\pi} S_{V}(\omega) d \omega}
$$

where $E\left[V_{1}^{+}\right]$and $S_{V}(\omega)$ are the total number of zerocrossing for velocity record and its PSD, respectively. The PSD of velocity record is related to the PSD of acceleration record, $S_{A}(\omega)$, via:

$$
S_{V}(\omega)=\frac{S_{A}(\omega)}{\omega^{2}} .
$$

Even though this is obtained for the stationary signals, assuming the slow-varying conditions for the nonstationary signals, this formula can as well be extended to include those cases:

$$
S_{V}(\omega, t)=\frac{S_{A}(\omega, t)}{\omega^{2}}
$$

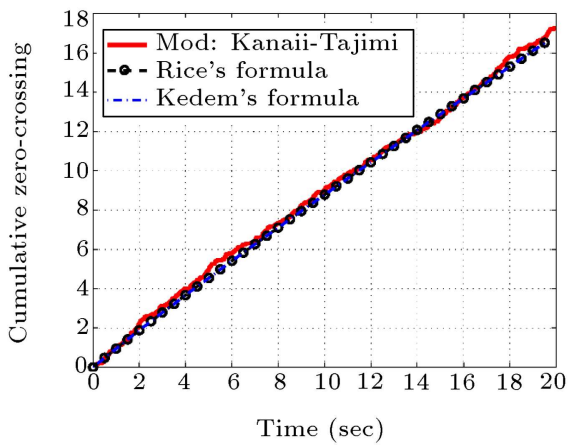

(a)

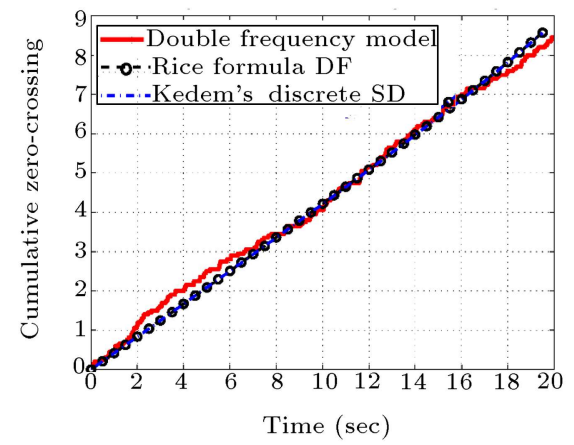

(c) where $S_{A}(\omega, t)$ represents the EPSD of the acceleration signal. It should be emphasized that for evolutionary process, $Y(t)$, defined by the Priestley's formula, the time derivative of the process, i.e., $Y(t)$, can also be described using Eq. (9); but, the corresponding modulating function, $\hat{m}(t, \omega)$, can be proved to have the following relationship with $m(t, \omega)$ [45]:

$$
\hat{m}(t, \omega)=\dot{m}(t, \omega)+(i \omega) m(t, \omega),
$$

where $\dot{m}(t, \omega)$ is the rate of the evolution of $m(t, \omega)$ with time. Since used in Eq. (14) projects the characteristics of $m(t, \omega)$ in the model introduced here, $H(\omega, t)$ used in Eq. (14) projects the characteristics of $m(t, \omega)$, as long as $\dot{m}(t, \omega)$ is small compared to $(i \omega) m(t, \omega)$ term, the EPSD of the time derivative of any process $\dot{Y}(t)$ can be estimated as $|H(\omega, t)|^{2} \omega^{2} d \mu(\omega)$ which is equal to $\omega^{2} S_{Y}(t, \omega)$. Therefore, using numerical or analytical evaluation, one can determine the validity of the slowvarying assumption by comparing the values of $\dot{H}(\omega, t)$ and $H(\omega, t)(i \omega)$.

The zero crossings of the velocity records result from using the EPSD of a high-pass filtered, and DFM of Eq. (16) in a LTV system represented by Eq. (15) is assessed to investigate the validity of the proposed equations. Figure 2 shows the efficiency of the proposed

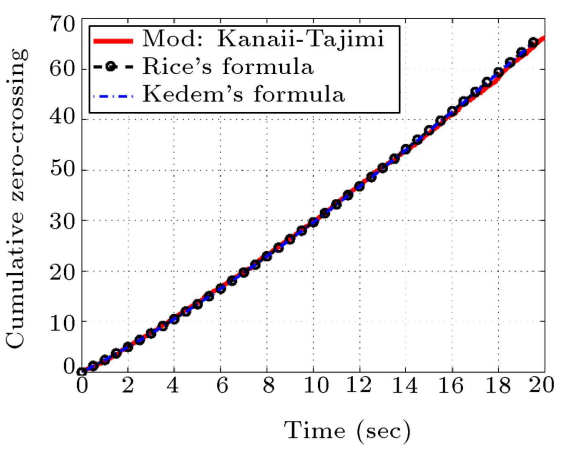

(b)

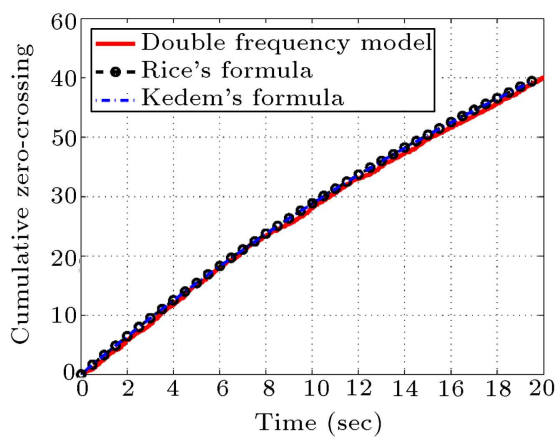

(d)

Figure 2. Comparison of the zero-crossing number for the simulated and estimated velocity time histories using the Rice (Eq. (22)) or Kedem's formula (Eq. (35)): (a) Zero crossing for $\xi_{g}=13 \%, F_{g}=2.14 \mathrm{~Hz}, F_{f 0}=3.89 \mathrm{~Hz}, F_{f n}=9.28 \mathrm{~Hz}$, $\xi_{f 0}=21 \%, \xi_{f n}=10 \%, \xi_{c}=7 \%, F_{c}=0.41 \mathrm{~Hz},(\mathrm{~b})$ zero crossing for $\xi_{g}=47 \%, F_{g}=5.39 \mathrm{~Hz}, F_{f 0}=2.91 \mathrm{~Hz}, F_{f n}=8.61$ $\mathrm{Hz}, \xi_{f 0}=44 \%, \xi_{f n}=26 \%, \xi_{c}=73 \%, F_{c}=0.86 \mathrm{~Hz},(\mathrm{c})$ zero crossing for $\xi_{g}=37 \%, F_{g}=1.59 \mathrm{~Hz}, F_{f 0}=8.04 \mathrm{~Hz}$, $F_{f n}=4.14 \mathrm{~Hz}, \xi_{f 0}=73 \%, \xi_{f n}=13 \%, \xi_{c}=28 \%, F_{c}=0.09 \mathrm{~Hz}$, and (d) zero crossing for $\xi_{g}=11 \%, F_{g}=5.8 \mathrm{~Hz}$, $F_{f 0}=4.51 \mathrm{~Hz} F_{f n}=2.85 \mathrm{~Hz}, \xi_{f 0}=17 \%, \xi_{f n}=13 \%, \xi_{c}=47 \%, F_{c}=0.25 \mathrm{~Hz}$. 
relation in computing the zero crossing of a velocity record calculated using Rice (Eq. (22)) or Kedem's formula (Eq. (35)) versus Monte Carlo simulations. As it is apparent, there is good agreement between the simulated zero crossings and the corresponding values determined by Kedem or Rice's formula.

The same procedure can be used to find the zero crossing of the displacement records by substituting the PSD of the displacement record in Eq. (35):

$$
\cos \left(\frac{2 \pi E\left[D_{1}^{+}\right]}{N-1}\right)=\frac{\int_{-\pi}^{\pi} \cos (\omega) S_{D}(\omega) d \omega}{\int_{-\pi}^{\pi} S_{D}(\omega) d \omega},
$$

where $E\left[D_{1}^{+}\right]$and $S_{D}(\omega)$ are the total number of zerocrossing for velocity record and its PSD, respectively. The forgoing procedure of estimating velocity and displacement zero-crossing for LTV systems was applied to the DFM, and Non-Stationary Modified KanaiTajimi (NSMKT) model was introduced by Waezi and Rofooei [38]. Figure 3 displays the difference between the zero-crossing of the Monte Carlo simulations and those estimated by Rice (Eq. (22)) or Kedem's (Eq. (39)) formulas. There is good agreement between the zero-crossing estimation by Kedem's or
Rice's formulas and the simulated records. Part of the differences between the Monte Carlo simulations and the analytic results is due to initial conditions used in the simulations while not being considered in the analytic calculations.

For both cases, the resulting zero crossing of a velocity time history fairly matches with those obtained from Monte Carlo simulations. Even though the displacement time history zero crossing obtained for DFM is in good agreement with those of Monte Carlo simulation, but in the case of NSMKT model, the differences are significant. The main reasons for the differences between the proposed formulas based on Kedem or Rice's relation with those obtained by simulations include: 1) the smaller duration of the simulated records; 2) normalization of the acceleration record's standard deviation to one; and 3 ) the variation rate of the model parameters. With regard to the first reason, since the IRF obtained from EPSD has infinite duration while there is no excitation for $t<0$; therefore, for the beginning part of the records, the output process will have a transient phase arising from zero initial condition. For an LTV system, it can be stated that:

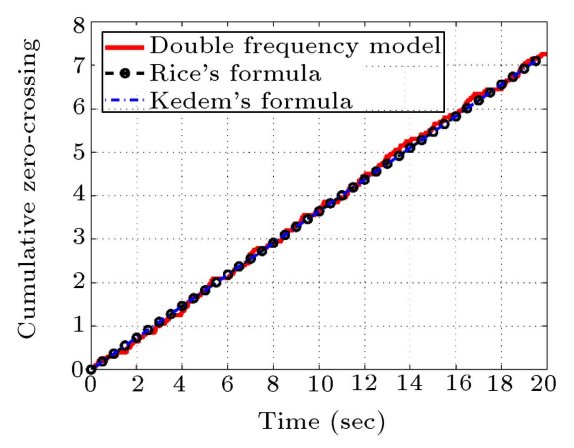

(a)

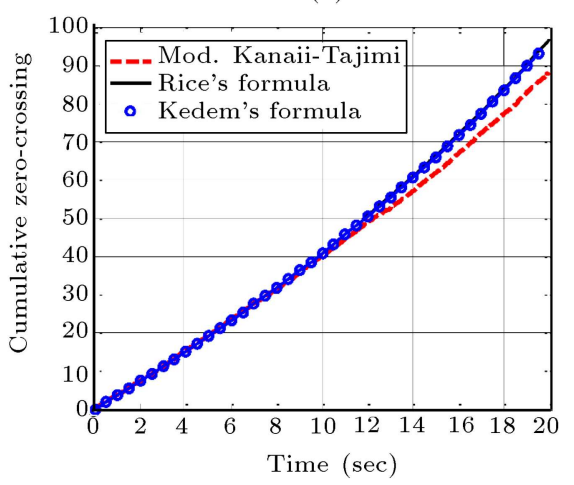

(c)

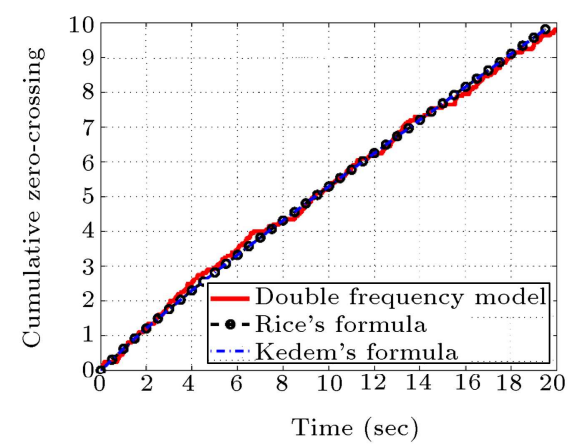

(b)

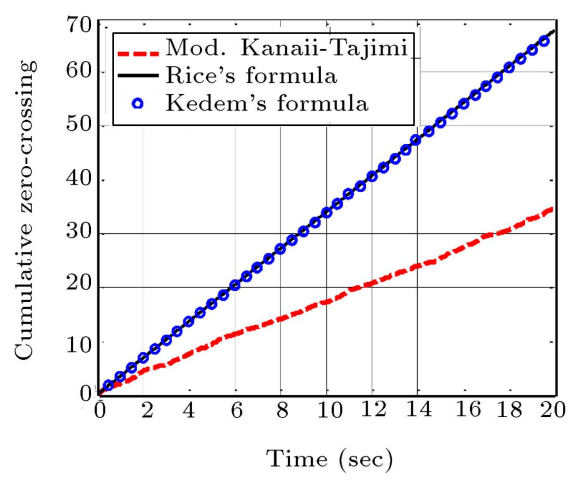

(d)

Figure 3. Comparison of the zero-crossing number for the simulated and estimated displacement time histories for ensembles corresponding to specific model parameters: (a) Displacement zero crossing $\xi_{g}=49 \%, F_{g}=0.6 \mathrm{~Hz}, F_{f 0}=9.67$ $\mathrm{Hz}, F_{f n}=6.36 \mathrm{~Hz}, \xi_{f 0}=65 \%, \xi_{f n}=61 \%, \xi_{c}=24 \%, F_{c}=0.37 \mathrm{~Hz}$, (b) displacement zero crossing $\xi_{g}=51 \%, F_{g}=1.24 \mathrm{~Hz}$, $F_{f 0}=1.17 \mathrm{~Hz}, F_{f n}=6.59 \mathrm{~Hz}, \xi_{f 0}=18 \%, \xi_{f n}=81 \%, \xi_{c}=55 \%, F_{c}=0.45 \mathrm{~Hz},(\mathrm{c})$ displacement zero crossing $\xi_{g}=52 \%$, $F_{g}=4.05 \mathrm{~Hz}, F_{f 0}=5.07 \mathrm{~Hz}, F_{f n}=8.66 \mathrm{~Hz}, \xi_{f 0}=67 \%, \xi_{f n}=11 \%, \xi_{c}=60 \%, F_{c}=0.50 \mathrm{~Hz}$, and (d) displacement zero crossing $\xi_{g}=45 \%, F_{g}=4.43 \mathrm{~Hz}, F_{f 0}=5.69 \mathrm{~Hz}, F_{f n}=5.69 \mathrm{~Hz}, \xi_{f 0}=73 \%, \xi_{f n}=73 \%, \xi_{c}=10 \%, F_{c}=0.30 \mathrm{~Hz}$. 


$$
\begin{aligned}
a(n) & =\sum_{k=0}^{n-1} w(k) h(n-k, k) \\
& =\sum_{k=-\infty}^{\infty} U(k) w(k) h(n-k, k),
\end{aligned}
$$

where $w(k), U(k)$, and $a(n)$ represent the ideal discrete white noise, unit-step function, and the corresponding output acceleration process, respectively. The evolutionary Discrete-Time Fourier Transform (DTFT) can be defined as follows:

$$
\begin{aligned}
A(\omega, n)= & \sum_{j=0}^{n-1} a(j) e^{-i \omega j} \\
= & \sum_{j=-\infty}^{\infty} \sum_{k=-\infty}^{\infty} \operatorname{Rect}(j, n) U(k) w(k) h(j \\
& -k, k) e^{-i \omega(j)}=\sum_{k=-\infty}^{+\infty} U(k) w(k) e^{-i \omega k} \\
& \sum_{j=-\infty}^{+\infty} \operatorname{Rect}(j, n) h(j-k, k) e^{-i \omega(j-k)} \\
= & \sum_{k=-\infty}^{\infty} U(k) w(k) e^{-i \omega k} \hat{H}_{n}(\omega, k)
\end{aligned}
$$

where $\hat{H}_{n}(\omega, k)$ is $\sum_{j=0}^{n-1} h(j-k, k) e^{-i \omega(j-k)}$ and represents the result of DTFT of multiplication of a truncation window, $R(j, n)$, with $n$-size width and the IRF represented by $h(j-k, k)$. It is obvious that as $n$ gets larger, $\hat{H}_{n}(\omega, k)$ approaches the prescribed evolutionary transfer function, $H(\omega, k)$, defined by Eq. (14) as follows:

$$
H(\omega, k)=\sum_{j=-\infty}^{+\infty} h(j-k, k) e^{-i \omega(j-k)} .
$$

However, for small values of $n, \hat{H}_{n}(\omega, k)$ is obtained through the convolution integral of two DTFTs corresponding to the $n$-sized rectangle window and the IRF which changes the frequency content of the IRFs up to the considered time. Figure 4 illustrates that as the size of the symmetric truncation window decreases, the output Fourier Transform deviates from the ideal Dirac delta, $\delta(\omega)$, and some energy leaks to the frequencies other than zero. Due to the procedure of truncation, the low-frequency part of the EPSD for the early part of the output process is accentuated, and its reflection on the displacement record's power spectrum is intensified. Using the EPSD definition, it can be concluded that:

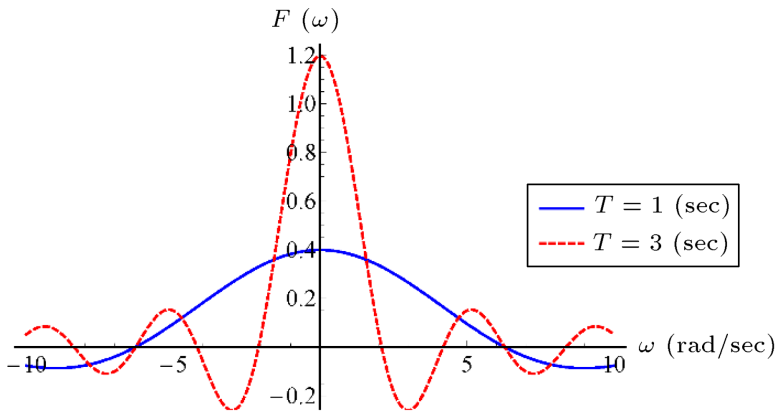

Figure 4. The Fourier transform of the rectangular truncation windows with different widths.

$$
|A(\omega, n)|^{2}=\sigma_{w}^{2} \sum_{k=0}^{n}\left|\hat{H}_{k}(\omega, k)\right|^{2},
$$

where $\sigma_{w}$ is the standard deviation of the input white noise.

For the second reason, the normalization of the variance of the output signal from LVT system changes the zero crossing of the displacement signal. This is due to the fact that multiplication of the output signal by $q(t) / \sigma(t)$ will affect the low-frequency part of the power spectrum. By means of the relationship between convolution output, $a(t)$, and the resulted normalized record, $\hat{a}(t)^{\prime}$ :

$$
\hat{a}(t)=\frac{1}{e(t)} a(t),
$$

where $e(t)=\frac{\sigma(t)}{q(t)}$, one can estimate the zero-crossing rate as follows:

$$
Z C_{\hat{a}}=\frac{\sigma_{\hat{a}}}{\sigma_{\hat{a}}}=\sqrt{Z C_{a}^{2}-\frac{\dot{e}(t)^{2}}{e(t)^{2}}} .
$$

It is seen that while $\frac{\dot{e}(t)^{2}}{e(t)^{2}}$ term is negligible for a smooth $\sigma(t)$, there is no much difference between $Z C_{\hat{a}}$ and $Z C_{a}$. However, the effect of multiplication by $1 / e(t)$ may be significant on the displacement signal since it is highly sensitive to the low-frequency content. Regarding the third reason, the violation of slowvarying model parameters leads to the overestimation of the displacement record zero crossing for NSMKT model, as clearly seen in Figure 5.

To address this problem, the following analytic equation for estimating $\sigma(t)$ of $a(n)=\sum_{k=0}^{n} w(k) h(n-$ $k, k)$ is considered:

$$
\begin{gathered}
\sigma(n)^{2}=E\left[a(n)^{2}\right]=\sum_{k=0}^{n} \sum_{j=0}^{n} w(k) w(j) h(n-j, j) h \\
(n-k, k)=\sigma_{w}^{2} \sum_{k=0}^{n} h^{2}(n-k, k) .
\end{gathered}
$$

It can be claimed that using this analytic equation 


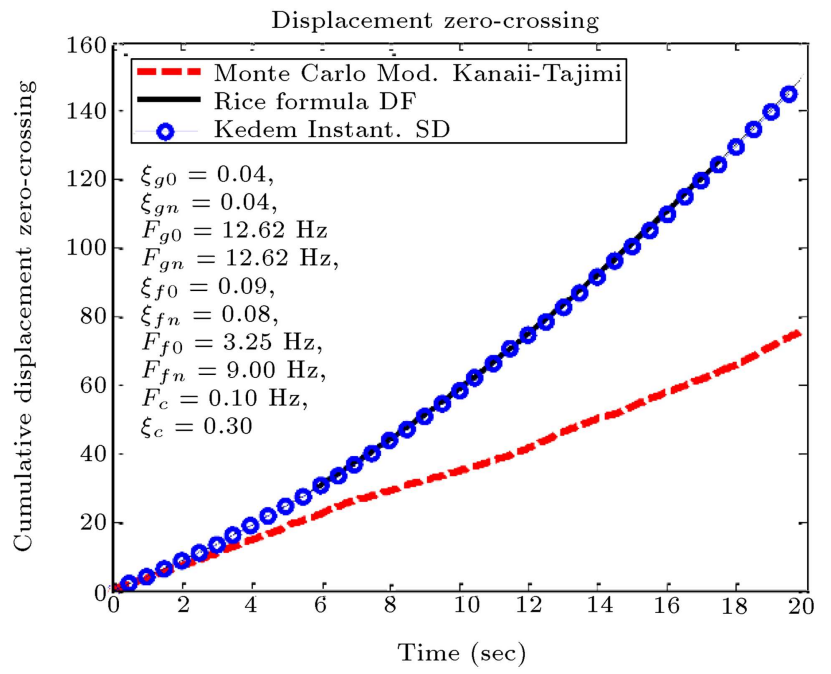

Figure 5. The overestimation of the displacement record by analytic formula due to the normalization process.

instead of estimating the zero-crossing rate of the displacement record directly from the normalized record, one can extract $a(n)$ from the initial record with multiplication of the target record by $\sigma(n) / q(n)$, in which $q(n)$ is the amplitude envelope of the original record.

The efficiency of the proposed method in determining the zero-crossings of the displacement records is investigated by examining the output signals obtained from an LTV system with the NSMKT model defined by Eq. (16). Monte Carlo simulations based on 5000 sets of model parameters, $\left\{\omega_{g}, \xi_{g}, \omega_{f_{0}}, \omega_{f_{n}}, \xi_{f_{0}}, \xi_{f_{n}}\right\}$, are performed with 20 realizations for each set. Table 2 summarizes the methods used for the evaluation of the displacement records of zero crossing based on the proposed method. The second column of the table indicates if the Rice or Kedem's formula (Eq. (22) or Eq. (19)) has been employed in each method or not. The third column describes the EPSD used in the Rice or Kedem's formula which may be the analytic EPSD of Eq. (16) or real-time evaluated EPSD through autocorrelation function of the record. The forth column indicates whether the instantaneous or averaged EPSD (up to the desired time) of the record has been used for the evaluation of the displacement zero-crossing. The fifth column determines if the methods use the modifications described by Eq. (43) to get adjusted to the effects of the truncation. In order to consider the effect of the normalization of the output signal on the desired modulating function, one method uses the convolution integral of the PSD for $1 / e(t)$ and $a(t)$ in Eq. (44). Figure 6 shows the average error for 5000 cases of the simulation between the simulated and semi-analytically calculated displacement records of zero-crossings for each method. As seen, using the methods, which do not consider the truncation error, leads to large estimation error. On the other hand, even though the improved methods yield better results, they do not necessarily end up with the perfect estimation of the displacement time history's crossing. The overall assessments indicate that impressive results have been achieved by "ZC Kedem Truncated" with an average error of $15 \%$, while it does not include the normalization effect and improves the performance of the original Kedem formula by reducing its average error by at least $70 \%$. The undesirable results of the "ZC Kedem Truncated Env." method that considers the effect of the normalization are due to the fact that the outcome EPSD of the multiplication of two signals cannot be estimated by the convolution of their respective EPSDs due to the missing phase information of the signals. It seems that the contribution of the normalization of the output to a desired modulating function is of lower importance compared with that caused by the truncation issue.

\section{Simulation and results}

Having defined the 4 time-domain measures of frequency Non-Stationarity (NS) (acceleration, velocity, and displacement time history records' zero-crossings

Table 2. The methods used for the evaluation of the displacement zero crossing.

\begin{tabular}{|c|c|c|c|c|c|}
\hline Method & Equation & $\begin{array}{l}\text { PSD } \\
\text { used }\end{array}$ & $\begin{array}{c}\text { Averaged or } \\
\text { instantaneous } \\
\text { PSD }\end{array}$ & $\begin{array}{c}\text { Truncation } \\
\text { effect }\end{array}$ & $\begin{array}{c}\text { Envelope } \\
\text { function } \\
\text { effect }\end{array}$ \\
\hline ZC Rice & Rice & Disp. HPF PSD & Instantaneous & No & No \\
\hline ZC Kedem & Kedem & Disp. HPF PSD & Instantaneous & No & No \\
\hline ZC Kedem mean autocorrelation & Kedem & Autocorrelation PSD & Instantaneous & No & No \\
\hline ZC Kedem mean displacement PSD & Kedem & Disp. HPF PSD & Averaged & No & No \\
\hline ZC Kedem instantaneous displacement & Kedem & Disp. HPF PSD & Instantaneous & No & No \\
\hline ZC Rice mean autocorrelation & Rice & Autocorrelation PSD & Instantaneous & No & No \\
\hline ZC Kedem truncation modified & Kedem & Disp. HPF PSD & Averaged & Yes & No \\
\hline ZC Kedem truncation \& envelope modified & Kedem & Disp. HPF PSD & Averaged & Yes & Yes \\
\hline
\end{tabular}




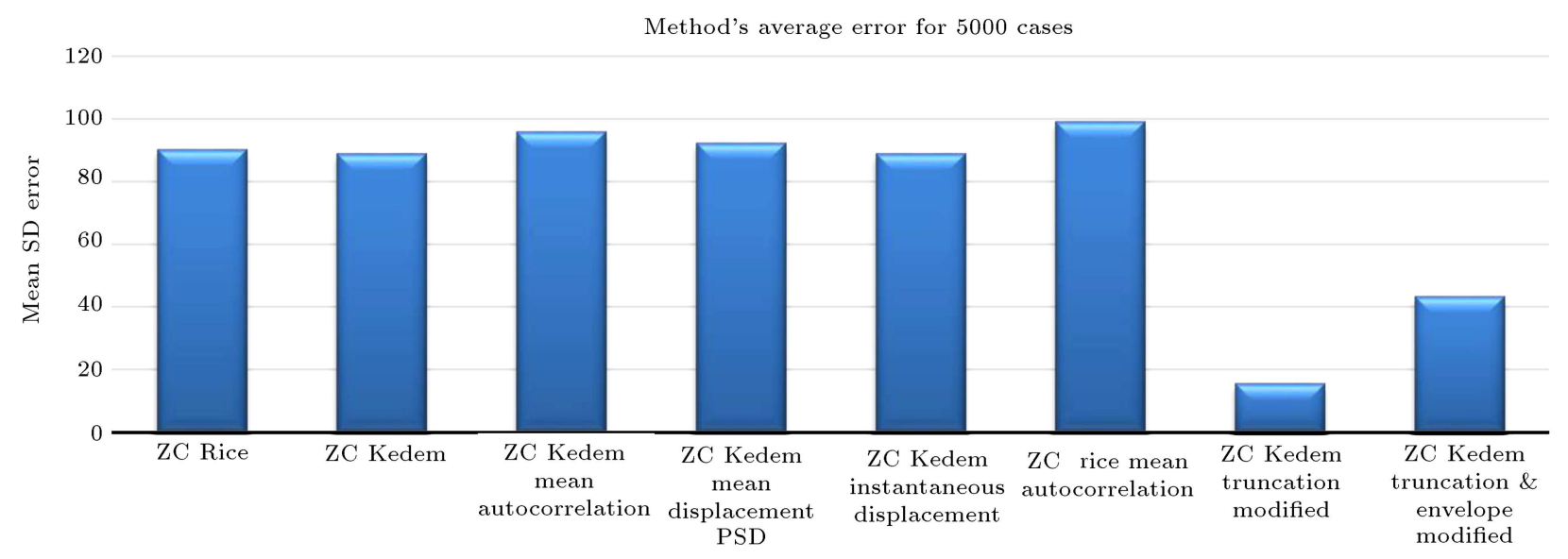

Figure 6. The average error between the simulated displacement zero-crossing and the values obtained from the proposed semi-analytic methods.

as well as acceleration pos. min./neg. max.) independent of the Amplitude Non-Stationarity (ANS), it is now possible to propose a novel algorithm for the generation of the NS acceleration records for a "target" record. In that regard, an appropriate EPSD is adopted for which IRF is determined by inverse Fourier transform. According to the four time-domain measures, an objective function consisting of the sum of the squares of the difference between simulated and seed records is defined which can be minimized using any optimization tool by adjusting model parameters. Having obtained the IRF model parameters and taking advantage of independency of FNST measures from ANST, any amplitude modulating function may be selected from Table 1.

Waezi and Rofooei [38] used the NSTMKT model along with only acceleration record's zero crossing and pos. min./neg. max. to simulate 106 near-field pulseless records with $R_{\text {rup }}$ less than $10 \mathrm{~km}$. The optimized model's IRF parameters, $\omega_{g}, \xi_{g}, \omega_{f_{0}}, \omega_{f_{n}}, \xi_{f_{0}}, \xi_{f_{n}}$, are determined according to the minimization of the following objective function:

$$
\begin{gathered}
\mathbf{F}\left(\omega_{g}, \xi_{g}, \omega_{f_{0}}, \omega_{f_{n}}, \xi_{f_{0}}, \xi_{f_{n}}\right)=\sum\left(P M N M_{\text {sim }}\right. \\
\left.-P M N M_{\text {Target }}\right)^{2}+\sum\left(Z C_{\text {sim }}-Z C_{\text {Target }}\right)^{2}
\end{gathered}
$$

In the above equation, $P M N M$ and $Z C$ stand for the cumulative counts of the pos. min. and neg. max. and zero crossing of the acceleration process, respectively. The "sim" and "target" subscripts represent the obtained values of the simulated and the target records, respectively. The synthetic records are simulated according to Eq. (15) where $q(t)$ is assumed to be the piece-wise modulating function of Table 1 . The appropriate modulating function's parameters are determined in a way that their Arias intensity curve fits the original's record effectively.
The compatibility of the output records is investigated using the difference between spectral displacements and zero crossing as well as pos. min./neg. $\max$ [38]. Even though the simulations were satisfactory for the low-period region of the response spectrum, the outcome does not indicate such a proper long-period spectral compatibility. A part of this outcome may stem from the fact that the utilized criteria for the estimation of the IRF parameters do not take into account the low-frequency content of the PSD. To overcome this problem, the same simulations are repeated here, but the four compatibility criteria mentioned in Section 4 are used to track the evolution of the EPSD in time for both high and low-frequency regions of the spectrum. The proposed approach capable of considering truncation issue in the estimation of the displacement record zero crossings has been utilized in the process of target record simulations. Figure 7 compares the empirical distribution of the spectral displacement error for the simulation of 106 near-field pulse-less records used by Waezi and Rofooei [38] as seed records. Two curves in this figure show the results

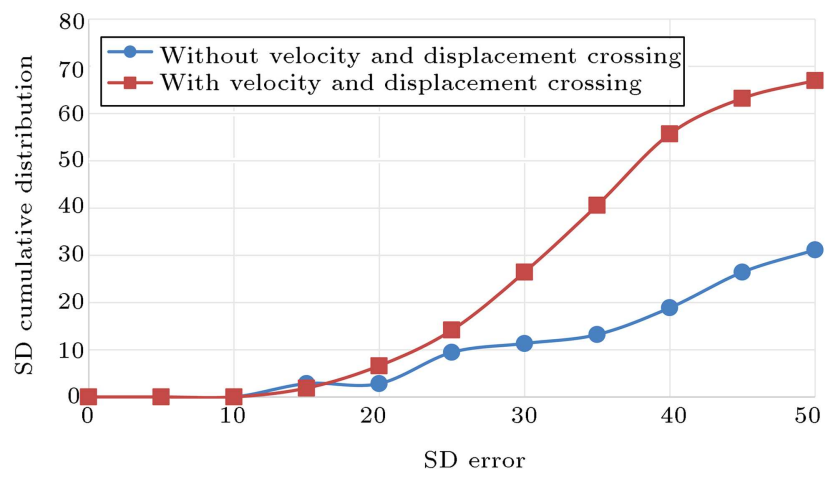

Figure 7. The comparison of the empirical distributions of the average Spectral Displacement (SD) error of 106 near-field pulse-less records simulation with/without considering the velocity and displacement crossing compatibility. 
of the simulation based on methods that are similar in considering accelerations record's zero crossing and pos. min./neg. max. counts, but different in considering velocity and displacement records' zero crossings in the simulation process. The results indicate that via contemplating the effect of low-frequency spectrum by incorporating the displacement and velocity zero crossings into the simulation process, the compatibility of the resulted non-stationary records with the seed records has been drastically improved. This outcome provides the evidence for the necessity of including the low-frequency-related indices, such as low-level crossings in the simulation of the non-stationary records based on LTV systems. It is also worthy to notice that only time-domain characteristics of the target records are used to evaluate the model parameters' results for proper response spectrum compatibility of the simulated records with the target one. In other words, the results of $[9,18,38]$ show that finding the model's parameters to satisfy the compatibility of the acceleration processes' zero-crossing and pos. min. \& neg. max. ensures the compatibility of the shortperiod region of the response spectrum. But, in order to get acceptable response spectrum match in the longperiod region, other indices should be added, such that velocity and displacement's zero crossings are shown to be the best candidates to do so. This is an indication that instead of tracking the EPSD or the response spectrum matching, one can use the time-domain EPSD-related indices of the energy content evolution, and these criteria could lead to proper agreement in the response spectrum of the simulated records with those of target record. A similar notion of tracking the non-stationarity of the ground-motion by means of time-domain indices is also used by Rezaeian et al. [46] to track the nonstationary characteristics of the 1994 Norhtridge earthquake records.

\section{Conclusion}

This paper introduces a new method for investigating the evolutionary spectrum of the output signals from LTV systems with time-varying IRF. Using the assumption of semi-stationarity, the analytic expressions describing zero crossing and pos. min. /neg. max. cumulative curves are derived for the NST signals with known EPSD resulted from LTV systems subjected to white-noise input. Due to the insufficiency of these two measures for proper tracking of the low-frequency energy contents, the velocity and displacement zero crossings of the output signals are suggested as further criteria of EPSD compatibility. Considering the common issues concerning the evaluation of these two measures, the analytic method of estimation is introduced and their drawbacks are discussed using the results obtained from 5000 Monte
Carlo simulations. Finally, a general algorithm to generate the earthquake acceleration records according to a seed record is suggested using LTV systems and the aforementioned four time-domain measures. The proposed algorithm is applied to simulate 106 near-filed pulse-less acceleration records, and the results indicate the improved compatibility of the generated records with the target one because of the four-part objective function and the modification to the displacement zerocrossing estimation.

\section{Acknowledgment}

The authors gratefully acknowledge the helpful comments of Dr. Yousef Bozorgnia from University of California at Los Angeles in completion of this study. Also, special thanks are due to Dr. Sanaz Rezaeian from U.S. Geological Survey for providing the authors with model software and helpful discussions.

\section{References}

1. Bommer, J.J. and Acevedo, A.B. "The use of real earthquake accelerograms as input to dynamic analysis", J. Earthquake Eng., 8(spec01), pp. 43-91 (2004).

2. Kramer, S.L., Geotechnical Earthquake Engineering, 80, Prentice Hall Upper Saddle River, NJ (1996).

3. Kostić, S., Vasović, N., Perc, M., Toljić, M. and Nikolić, D. "Stochastic nature of earthquake ground motion", Physica A: Statistical Mechanics and Its Applications, 392(18), pp. 4134-4145 (2013).

4. Douglas, J. and Aochi, H. "A survey of techniques for predicting earthquake ground motions for engineering purposes", Surv. Geophys., 29, pp. 187-220 (Oct. 2008).

5. Alamilla, J., Esteva, L., Garcıa-Perez, J. and DıazLopez, O. "Evolutionary properties of stochastic models of earthquake accelerograms: Their dependence on magnitude and distance", J. Seismol., 5, pp. 1-21 (2001).

6. Hartzell, S., Frankel, A., Liu, P., Zeng, Y. and Rahman, S. "Model and parametric uncertainty in source-based kinematic models of earthquake ground motion", Bull. Seismol. Soc. Am., 101(5), pp. 24312452 (2011).

7. Motazedian, D. and Atkinson, G. "Stochastic finitefault modeling based on a dynamic corner frequency", Bull. Seismol. Soc. Am., 95(3), pp. 995-1010 (2005).

8. Boore, D.M. "Simulation of ground motion using the stochastic method", Pure Appl. Geophys., 160(3-4), pp. 635-676 (2003).

9. Rezaeian, S. and Der Kiureghian, A. "A stochastic ground motion model with separable temporal and spectral nonstationarities", Earthquake Eng. Struct. Dyn., 37(13), pp. 1565-1584 (2008). 
10. Stafford, P., Sgobba, S. and Marano, G. "An energybased envelope function for the stochastic simulation of earthquake accelerograms", Soil Dyn. Earthquake Eng., 29, pp. 1123-1133 (2009).

11. Sun, X., Hartzell, S. and Rezaeian, S. "Ground-motion simulation for the 23 August 2011, mineral, Virginia, earthquake using physics-based and stochastic broadband methods", Bull. Seismol. Soc. Am., 105(5), pp. 2641-2661 (2015).

12. Campbell, K.W. "Prediction of strong ground motion using the hybrid empirical method and its use in the development of ground-motion (attenuation) relations in eastern North America", Bull. Seismol. Soc. Am., 93(3), pp. 1012-1033 (2003).

13. Conte, J.P. and Peng, B.F. "Nonstationay earthquake ground motion model", 11th World Conference on Earthquake Engineering, Acapulco, Mexico (1996).

14. Yeh, C.H. and Wen, Y.K. "Modeling of nonstationary ground motion and analysis of inelastic structural response", Struct. Saf., 8, pp. 281-298 (1990).

15. Kiureghian, A.D. and Crempien, J. "An evolutionary model for earthquake ground motion", Struct. Saf., 6, pp. 235-246 (1989).

16. Yamamoto, Y. and Baker, J.W. "Stochastic model for earthquake ground motion using wavelet packets", Bull. Seismol. Soc. Am., 103(6), pp. 3044-3056 (2013).

17. Yang, D. and Yang, K. "Multi-step prediction of strong earthquake ground motions and seismic responses of SDOF systems based on EMD-ELM method", Soil Dyn. Earthquake Eng., 85, pp. 117-129 (2016).

18. Rezaeian, S. and Der Kiureghian, A. "Simulation of synthetic ground motions for specified earthquake and site characteristics", Earthquake Eng. Struct. Dyn., 39(10), pp. 1155-1180 (2010).

19. Gabor, D. "Theory of communication. Part 1: The analysis of information", Journal of the Institution of Electrical Engineers-Part III: Radio and Communication Engineering, 93(26), pp. 429-441 (1946).

20. Huang, N.E., Shen, Z., Long, S.R., Wu, M.C., Shih, H.H., Zheng, Q., et al. "The empirical mode decomposition and the Hilbert spectrum for nonlinear and non-stationary time series analysis", Proceedings of the Royal Society of London A: Mathematical, Physical and Engineering Sciences, pp. 903-995 (1998).

21. Huang, N.E., Wu, Z., Long, S.R., Arnold, K.C., Chen, $\mathrm{X}$. and Blank, K. "On instantaneous frequency", Adv. Adapt. Data Anal., 1(02), pp. 177-229 (2009).

22. Page, C.H. "Instantaneous power spectra", J. Appl. Phys., 23(1), pp. 103-106 (1952).

23. Priestley, M.B. "Evolutionary spectra and nonstationary processes", J. Roy. Stat. Soc. Ser. B. (Stat. Method.), 27(2), pp. 204-237 (1965).

24. Saragoni, G.R. and Hart, G.C. "Simulation of artificial earthquakes", Earthquake Eng. Struct. Dyn., 2(3), pp. 249-267 (1974).
25. Liu, S.-C. "Evolutionary power spectral density of strong-motion earthquakes", Bull. Seismol. Soc. Am., 60(3), pp. 891-900 (1970).

26. Amin, M. and Ang, A.H. "Nonstationary stochastic models of earthquake motions", J. Eng. Mech., 94(2), pp. 559-584 (1968).

27. Shinozuka, M. and Sato, Y. "Simulation of nonstationary random processes", J. Eng. Mech., 93(1), pp. 11-40 (1967).

28. Liang, J., Chaudhuri, S.R. and Shinozuka, M. "Simulation of nonstationary stochastic processes by spectral representation", J. Eng. Mech., 133(6), pp. 616-627 (2007).

29. Kaveh, A. and Mahdavi, V.R. "A new method for modification of ground motions using wavelet transform and enhanced colliding bodies optimization", Applied Soft Computing, 47, pp. 357-369 (2016).

30. Papadimitriou, K., Stochastic Characterization of Strong Ground Motion and Application to Structural Response, Pasadena, CA EERL, pp. 90-03 (1990).

31. Rice, S.O. Mathematical analysis of random noise", Bell Syst. Tech. J., 23(3), pp. 282-332 (1944).

32. Dugundji, J. "Envelopes and pre-envelopes of real waveforms", Information Theory, IRE Transactions on, 4(1), pp. 53-57 (1958).

33. Sgobba, S., Stafford, P. and Marano, G. "A seismologically consistent husid envelope function for the stochastic simulation of earthquake ground-motions", in Computational Methods in Stochastic Dynamics, 22, Papadrakakis, M., Stefanou, G., and Papadopoulos, V., Eds., pp. 229-246, Springer, Netherlands (2011).

34. Crandall, S.H. and Mark, W.D., Random Vibration in Mechanical Systems, Academic Press (2014).

35. Bolotin, V. "Statistical theory of the aseismic design of structures", Proc. Second World Conf. on Earthquake Engineering, Tokyo, pp. 1365-1374 (1960).

36. Arias, A., Holzapfel, A. and Saragoni, G.R. "An approximate expression for the mean square acceleration of earthquake ground motions", Fifth World Conference on Earthquake Engineering, Roorkee, India (1976).

37. Yeh, C.-H., Modeling of Nonstationary Earthquake Ground Motion and Biaxial and Torsional Response of Inelastic Structures, University of Illinois at UrbanaChampaign (1989).

38. Waezi, Z. and Rofooei, F.R. "Stochastic nonstationary model for ground motion simulation based on higher-order crossing of linear time variant systems", J. Earthquake Eng., 21(1), pp. 123-150 (2017).

39. Mobarakeh, A.A., Rofooei, F.R. and Ahmadi, G. "Simulation of Earthquake Records Using time-varying ARMA(2,1) model", Probabilist. Eng. Mech., 17(1), pp. 15-34 (2002).

40. Boashash, B. "Estimating and interpreting the instantaneous frequency of a signal. I. Fundamentals", Proc. IEEE, 80(4), pp. 520-538 (1992). 
41. Tsatsanis, M.K. and Giannakis, G.B. "Subspace methods for blind estimation of time-varying FIR channels", IEEE T. Signal. Proces., 45(12), pp. 3084-3093 (1997).

42. Rofooei, F., Mobarake, A. and Ahmadi, G. "Generation of artificial earthquake records with a nonstationary Kanai-Tajimi model", Eng. Struct., 23(7), pp. 827-837 (2001).

43. Kanasewich, E.R., Time Sequence Analysis in Geophysics, University of Alberta Press (1981).

44. Kedem, B. "Spectral analysis and discrimination by zero-crossings", Proc. IEEE, 74(11), pp. 1477-1493 (1986).

45. Michaelov, G., Sarkani, S. and Lutes, L. "Spectral characteristics of nonstationary random processesa critical review", Struct. Saf., 21(3), pp. 223-244 (1999).

46. Rezaeian, S., Zhong, P., Hartzell, S. and Zareian, F. "Validation of simulated earthquake ground motions based on evolution of intensity and frequency content", Bull. Seismol. Soc. Am., 105(6), pp. 3036-3049 (2015).

\section{Biographies}

Zakariya Waezi received his BSc degree in Civil and Petroleum Engineering from Sharif University of Technology, Iran, in 2007. He received his MSc degree in Earthquake Engineering in 2009. He is a PhD candidate in Structural Engineering at Sharif University of Technology.

Fayaz Rahimzadeh Rofooei is a Professor of Civil Engineering at Sharif University of Technology, Iran, since 2005. He received his BSc degree in Civil Engineering from Iran University of Science and Technology. He received his MSc degrees in Mathematics and Structural Optimization and also $\mathrm{PhD}$ degree in Civil Engineering (Structural Control) from Rensselaer Polytechnic Institute, Troy, N.Y., USA. His research interests are active and passive structural control, nonlinear structural analysis, base isolation, seismic vulnerability analysis and retrofitting of structures and lifelines. 